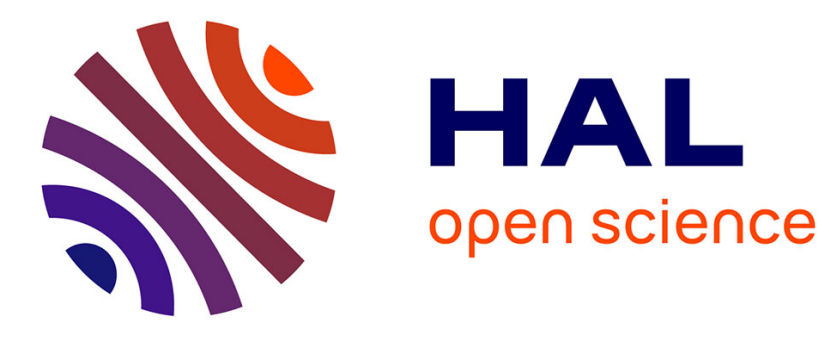

\title{
Thermographic Analysis of Material Behavior
}

Jean-Christophe Batsale, André Chrysochoos, Hervé Pron, Bertrand Wattrisse

\section{To cite this version:}

Jean-Christophe Batsale, André Chrysochoos, Hervé Pron, Bertrand Wattrisse. Thermographic Analysis of Material Behavior. Michel Grédiac; François Hild; André Pineau. Full-field measurements and identification in solid mechanics, John Wiley \& Sons, 2013, 978-1-84821-294-7. 10.1002/9781118578469.ch16 . hal-03338517

\section{HAL Id: hal-03338517 https://hal.science/hal-03338517}

Submitted on 8 Sep 2021

HAL is a multi-disciplinary open access archive for the deposit and dissemination of scientific research documents, whether they are published or not. The documents may come from teaching and research institutions in France or abroad, or from public or private research centers.
L'archive ouverte pluridisciplinaire HAL, est destinée au dépôt et à la diffusion de documents scientifiques de niveau recherche, publiés ou non, émanant des établissements d'enseignement et de recherche français ou étrangers, des laboratoires publics ou privés. 


\title{
Thermographic analysis of material behavior
}

\author{
J.-C. Batsale ${ }^{1}$, A. Chrysochoos ${ }^{2}$, H. Pron ${ }^{3}$, B. Wattrisse ${ }^{2}$ \\ 1. I2M, Arts et Métiers ParisTech, Bordeaux \\ 2. LMGC, Université Montpellier 2 \\ 3. GRESPI, Université de Reims Champagne-Ardenne
}

\subsection{Introduction}

Infrared techniques have been used to an increasing extent over the last 15 years in the field of Mechanics of Materials and Structures in university laboratories and industrial research and development centers. This has been facilitated by progress achieved in signal digitization, data transfer rates and image processing techniques, thus making new thermographic workstations more user-friendly. A key asset of thermographic systems is that they generate contactless full-field surface temperature measurements. In addition, current IR cameras allow the storage of thermal images of about 80000 pixels at frame rates of up to several hundred hertz, and even higher under certain conditions. New sensors enable the estimation of temperature variations ranging from several hundredths to several hundreds of degrees Celsius.

Thermal imaging is of interest in Mechanics, since material deformation is nearly always accompanied by heat release and thus temperature variations. Strain-induced heat sources have several origins - we shall distinguish sources associated with energy dissipation from those induced by thermomechanical coupling mechanisms. The first reflect the irreversibility of deformation processes. When the material behavior is plastic or viscoplastic, when ageing or damage processes occur, part of the deformation energy is irreversibly transformed into heat. The second, which are induced by thermomechanical couplings, also reveal the material behavior. They occur because the microstructural state is sensitive to temperature variations, inducing a strong interaction between the mechanical and thermal states. Thermoelasticity is surely the most famous example of coupling mechanisms [THO 53]. A thermoelastic material warms up under compression or expands when it is warmed. Of course, other coupling mechanisms exist. Another example concerns 


\section{IR thermographic imaging}

rubber elasticity [CHA 84] or mechanisms associated with austenite-martensite phase changes [PEY 98]. In the two latter cases, the materials cool under compression or contract when heated.

The quantitative assessment of evolved heat, caused by deformation, remains problematic despite progress achieved in the field of infrared data measurement, acquisition and processing. This analysis is based on measuring effects (temperature changes) to trace causes (release or stored heat). The relationship between temperature and source is complex because temperature changes depend both on the intensity and distribution of sources, but also on the nature of the material (diffusivity) and the thermal boundary conditions (external exchanges). Temperature variations are thus not definitely intrinsic to material behavior. Finally, the heat diffusion equation involves several thermophysical parameters (emissivity, specific heat, conduction tensor) that must be known in order to properly assess the temperature field and estimate the heat source distribution.

Infrared thermography applications in the Mechanics of Materials have primarily been focused on nondestructive testing. Thermal stress analysis is the oldest and most widespread application domain. Indeed, for a material having linear thermoelastic behavior (isotropic), surface temperature variations-with a magnitude of several tenths of a degree Celsius — can then be linked, with some additional assumptions, to variations in the stress tensor trace [SAT 07]. Another currently booming application area is the use of thermography to analyze the fatigue behavior of components. For monochromatic cyclic stress tests, the principle used to identify the most loaded zones of components is to analyze the time spectrum content of temperature fields, particularly the component associated with twice the loading frequency ( $2 f$ method [BRE 82]). This latter frequency generally does not correspond to thermoelastic effects and can be associated with the dissipative effects of fatigue. Self-heating ranges from a few degrees to several tens of degrees. Finally, we should also mention applications where temperature variations reach several tens to several hundreds of degrees. A few examples of this may be found in studies of material dynamic behavior [RAN 08], machining processes [SUT 07], forming and welding operations PIN [04], friction mechanisms, etc.

This chapter describes the main steps of a source field estimation protocol. It begins with a brief overview of the theoretical framework used to interpret thermal and calorimetric measurements and provides a link between heat sources and constitutive equations. Difficulties, associated with infrared measurements and their conversion in terms of temperature, are then discussed. Finally, various classic diffusion models linking temperature fields to heat source distributions, and allowing the estimation of thermo-physical parameters from thermal data, are introduced. 


\subsection{Thermomechanical framework}

Thermal measurements cannot be interpreted without reference to a specific thermodynamic framework, which is necessary for the definition of thermophysical parameters and the classification of heat sources. This section presents the framework of classic Thermodynamics of Irreversible Processes (TIP), whose applications to material behavior modeling began increasing in the 1960s. For further information, readers should refer to the work of [COL 67, HAL 75, GER 83] and more recently to [ENG 07]. TPI offers a consistent and flexible framework to describe material behavior. It is flexible since a set of state variables may be chosen to describe the macroscopic effects of numerous, complex and often combined microstructural phenomena.

\subsubsection{Constitutive equations}

The deformation process is considered to be quasi-static in a thermodynamic sense, i.e. consisting of a continuous series of equilibrium states. The equilibrium state of each elementary volume of matter is then described using a set of $N$ state variables. The temperature $T$, a strain tensor $\varepsilon$ and $N-2$ additional state variables are generally used to complete the description of the material state. $\alpha$ denotes the vector that symbolically groups these state variables, often called 'internal' state variables.

Let $\psi=e-T s$ define the Helmholtz specific free energy, where $e$ and $s$ are the internal energy and entropy, respectively. It is assumed that internal energy is a monotonically increasing function of entropy and absolute temperature is by definition $T=e_{\mathrm{s}}$.

The material constitutive equations are divided into two sets:

State equations: these are the first derivatives of the free energy with respect to state variables. These state laws read:

$$
\left\{\begin{array}{l}
s=-\psi_{, \mathrm{T}} \\
\sigma^{\mathrm{r}}=\rho \psi_{, \varepsilon} \\
A=\rho \psi_{, \alpha}
\end{array}\right.
$$




\section{IR thermographic imaging}

where $\sigma^{\mathrm{r}}$ is the reversible part of the stress tensor and $A$ a vector that groups the conjugated variables associated with internal state variables.

The local expression of the second principle of thermodynamics leads to the Clausius-Duhem inequality and allows the dissipation to be defined as:

$$
d=\sigma: D-\rho \psi_{, \varepsilon}: \dot{\varepsilon}-\rho \psi_{, \alpha} \cdot \dot{\alpha}-\frac{q}{T} \cdot \operatorname{grad} T \geq 0,
$$

where $\sigma$ is the Cauchy stress tensor, $D$ the Eulerian strain rate tensor, $\rho$ the mass density and $q$ the heat influx vector.

Evolution equations: in the particular context of Generalized Standard Materials [HAL 75], evolution equations are derived from the dissipation potential $\varphi(\dot{\varepsilon}, \dot{\alpha}, q ; T)$, depending on the flow of state variables, temperature $T$ (or any other state variable) acting as a parameter. The dissipation potential must be convex, positive, minimum and equal to zero in $(\dot{\varepsilon}=0, \dot{\alpha}=0, q=0)$ so that the ClausiusDuhem inequality can be verified during any thermodynamic process. When the dissipation potential is differentiable, the complementary behavioral equations can be formulated as:

$$
\left\{\begin{array}{ll}
-\frac{\operatorname{grad} T}{T}=\varphi_{, \mathrm{q}} & (a) \\
\sigma^{\mathrm{ir}}=\varphi_{, \dot{\varepsilon}} & (b) \\
X=\varphi_{, \dot{\alpha}} & (c)
\end{array} .\right.
$$

These define the thermodynamic forces and allow the dissipation to be reformulated as a sum of force-flux products. Under the small strain assumption, the strain rate tensor is identified with the time derivative of the strain tensor, and the dissipation becomes:

$$
d=\underbrace{\left(\sigma-\sigma^{\mathrm{r}}\right)}_{\sigma^{\mathrm{ir}}}: \dot{\varepsilon} \underbrace{-A}_{X} \cdot \dot{\alpha}-\frac{\operatorname{grad} T}{T} \cdot q \geq 0 .
$$

The identity $d \equiv 0$ defines a reversible thermodynamic process. Dissipation is usually written as the sum of two terms that are supposed to be separately positive and which are, in turn: the intrinsic dissipation, $d_{1}=\sigma^{\mathrm{ir}}: \dot{\varepsilon}+X . \dot{\alpha}$, characterizing material degradation, and thermal dissipation, $d_{2}=-\operatorname{grad} T / T . q$, reflecting the irreversible character of heat diffusion.

\subsubsection{Heat equation}


Under the small strain hypothesis, the two principles of thermodynamics can be locally written as:

$$
\left\{\begin{array}{l}
\rho \dot{e}=\sigma: \dot{\varepsilon}-\operatorname{div} q+r_{\mathrm{e}} \\
\rho T \dot{s}=d_{1}-\operatorname{div} q+r_{\mathrm{e}}
\end{array}\right.
$$

where $r_{\mathrm{e}}$ is the external heat supply (e.g. radiation energy). Once it is noted that

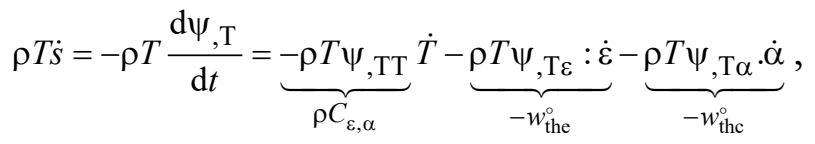

the local form of the heat diffusion equation can be directly deduced. Based on Fourier's conduction law, the heat equation can be written as follows:

$$
\rho C_{\varepsilon, \alpha} \dot{T} \underbrace{-\operatorname{div}(k \operatorname{grad} T)}_{q=-k \operatorname{grad} T}=\underbrace{d_{1}+T \sigma_{, \mathrm{T}}^{\mathrm{r}}: \dot{\varepsilon}+T A_{, \mathrm{T}} . \dot{\alpha}}_{w_{\mathrm{h}}^{\circ}}+r_{\mathrm{e}},
$$

where $C_{\varepsilon, \alpha}$ is the specific heat ${ }^{(1)}$ at constant $\varepsilon$ and $\alpha$, and $k$ is the conduction tensor.

The notation $(w)^{\circ}$ indicates that $(w)$ is dependent on the thermodynamic path followed. The term $w_{\mathrm{h}}^{\circ}$ stands for the overall heat rate accompanying the deformation process. The notation $w_{\mathrm{h}}^{\circ}$ means that $w_{\mathrm{h}}$ is not, a priori, a state function.

On the left-hand side of equation [16.7], the term $\rho C_{\varepsilon, \alpha} \dot{T}$ represents the heat rate stored or released per unit volume, while the term $-\operatorname{div}(k \operatorname{grad} T)$ corresponds to the local heat losses by conduction.

On the right-hand side, we successively find the intrinsic dissipation $d_{1}$, the heat sources associated with the thermomechanical coupling mechanisms (in brief, the coupling sources), taking into account the possible temperature sensitivity of reversible stress $\sigma^{\mathrm{r}}$ and conjugated variables $A$, and finally the external heat supply $r$.

1. The specific heat involved here is thus defined for isothermal processes during which all 
state variables except the temperature remain constant. The reader may question the relevance of the existence of such processes. Note also that the classic $C_{\mathrm{P}}$ found in Handbooks of Chemistry and Physics implies that temperature and pressure are the only state variables used to describe the thermomechanical behavior of a material.

The coupling sources can be divided into two parts: the thermoelastic source $w_{\text {the }}^{\circ}$, induced by material (thermo)dilatability, and other coupling sources $w_{\text {thc }}^{\circ}$, such as the latent heat rate of a first-order solid-solid phase change. It should be noted that, whatever the thermomechanical model, $w_{\text {the }}^{\circ}$ and $w_{\text {the }}^{\circ}$ do not correspond to $T \sigma_{\mathrm{T}}^{\mathrm{r}}: \dot{\varepsilon}$ and $T A_{\mathrm{r}} \cdot \dot{\alpha}$, respectively. The only general relationship that may be written is:

$$
w_{\text {the }}^{\circ}+w_{\text {the }}^{\circ}=T \sigma_{, \mathrm{T}}^{\mathrm{r}}: \dot{\varepsilon}+T A_{, \mathrm{T}} \cdot \dot{\alpha} .
$$

If $\rho$ et $C_{\varepsilon, \alpha}$ are material constants and if the conduction tensor also remains constant and isotropic, and where $k$ denotes the isotropic conduction coefficient, the heat equation can be simplified as:

$$
\rho C_{\varepsilon, \alpha} \dot{T}-k \Delta T=w_{\mathrm{h}}^{\circ}+r_{\mathrm{e}} .
$$

If we finally assume that the equilibrium temperature field $T_{0}$ and $r_{\mathrm{e}}$ are timeindependent, with $\theta=T-T_{0}$ denoting the temperature variation with respect to the local thermal equilibrium, then $-k \Delta T_{0}=r_{\mathrm{e}}$ and:

$$
\rho C_{\varepsilon, \alpha} \dot{\theta}-k \Delta \theta=w_{\mathrm{h}}^{\circ} .
$$

\subsubsection{Energy balance over a load-unload cycle}

Let us now consider a load-unload cycle, where $A=\left(T_{\mathrm{A}}, \varepsilon_{\mathrm{A}}, \alpha_{\mathrm{A}}\right)$ and $B=\left(T_{\mathrm{B}}, \varepsilon_{\mathrm{B}}, \alpha_{\mathrm{B}}\right)$ denote the thermomechanical states of the material at the extremities of the process. Let us then consider the energy balance corresponding to the three following situations:

- case $i: \mathcal{A} \neq \mathscr{B}$, this general situation can be illustrated by the schematic stress-strain diagram proposed in Fig. 1a.

- case $i i: A \neq \mathscr{B}$ and $\varepsilon_{\mathcal{A}}=\varepsilon_{\mathcal{B}}$, a mechanical cycle is then associated with the loadunload test. The stress-strain diagram shows a hysteresis loop.

- case $i i i: \mathcal{A}=\mathscr{B}$, the mechanical cycle is now a thermodynamic cycle. 


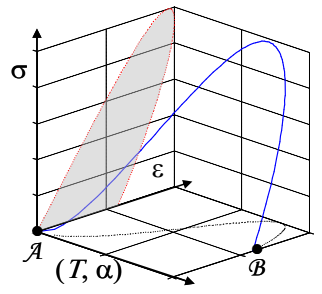

(i)

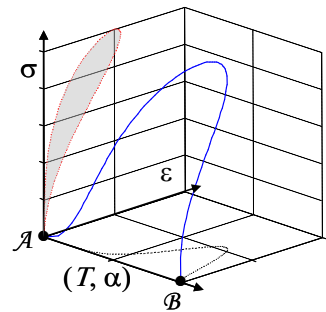

(ii)

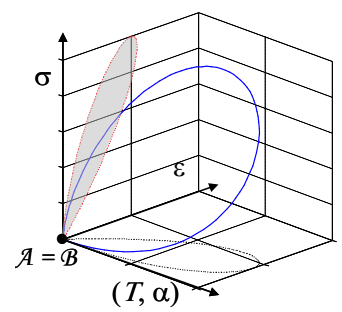

(iii)

Figure 16.1. Stress-strain curve constructed as the projection of a thermodynamic path onto the mechanical plane ([CHR 10]).

In the small strain framework, the deformation energy rate is defined by:

$$
w_{\mathrm{def}}^{\mathrm{o}}=\sigma: \dot{\varepsilon} .
$$

Eq.[16.4] shows that the intrinsic dissipation $d_{1}$ is then the difference between the deformation energy rate and the sum of the elastic and stored energy rates.

$$
\left\{\begin{array}{ll}
d_{1}=w_{\mathrm{def}}^{\circ}-\left(w_{\mathrm{e}}^{\circ}+w_{\mathrm{S}}^{\circ}\right) & (a) \\
w_{\mathrm{e}}^{\circ}+w_{\mathrm{S}}^{\circ}=\sigma^{\mathrm{r}}: \dot{\varepsilon}+A \cdot \dot{\alpha} & (b)
\end{array}\right. \text {. }
$$

Note again that the systematic correspondence between the two terms of equation $[16.12 \mathrm{~b}]$ is possible only for particular thermomechanical models.

Where $t_{\mathcal{B}}-t_{\mathcal{A}}$ denotes the duration of the test and based on equations [16.5] and [16.12], the deformation energy associated with a load-unload cycle may be formulated as follows:

$$
w_{\text {def }}=\int_{t_{\mathcal{A}}}^{t_{B}} d_{1} \mathrm{~d} t+\int_{t_{\mathcal{A}}}^{t_{\mathcal{B}}}\left(\rho \dot{e}-\rho C_{\varepsilon, \alpha} \dot{T}+w_{\text {the }}^{\circ}+w_{\text {thc }}^{\circ}\right) \mathrm{d} t
$$

where $e$ is the specific internal energy

Equation [16.13] shows that: 
- case $i$ : in the general case, the deformation energy balance during a load-unload test involves energy dissipation, internal energy variations (variations in heat stored in the material) and coupling heat sources.

- case $i i$ : the deformation energy then corresponds to the generalized area $A_{h}$ of the different hysteresis loops in planes $\sigma_{\mathrm{ij}}-\varepsilon_{\mathrm{ij}}, i, j=1,2,3$.

- case iii: for a thermodynamic cycle, these hysteresis loops are only due to dissipation and couplings, since the heat capacity $\rho C$ is assumed to be constant. The energy balance then takes the following simplified form:

$$
w_{\text {def }}=\int_{t_{\mathcal{A}}}^{t_{\mathcal{B}}}\left(\sum_{\mathrm{i}, \mathrm{j}=1}^{3} \sigma_{\mathrm{ij}} \dot{\varepsilon}_{\mathrm{ij}}\right) \mathrm{d} t=\int_{t_{\mathcal{A}}}^{t_{\mathcal{B}}} d_{1} \mathrm{~d} t+\int_{t_{\mathcal{A}}}^{t_{\mathcal{B}}}\left(w_{\text {the }}^{\circ}+w_{\text {thc }}^{\circ}\right) \mathrm{d} t .
$$

The energy balance then gives the restricted conditions for which the dissipated energy can be estimated by computing the hysteresis area of a uniaxial load-unload cycle. Indeed, Eq. [16.13] shows that coupling sources or internal energy variations can also influence the size of the hysteresis area. This underlines the necessity of analyzing the thermal effects to verify if a mechanical cycle is also a thermodynamic cycle, and to check the relative energy importance of coupling effects [CHR 10].

\subsection{Metrological considerations}

Infrared radiation corresponds to the electromagnetic spectrum ranging from 0.8 to $1000 \mu \mathrm{m}$ wavelengths. Infrared thermography generally uses short-wave (SW) and long-wave (LW) bands associated with the spectral domains 2-5 $\mu \mathrm{m}$ and 7-15 $\mu \mathrm{m}$, respectively. Radiation from the observed thermal scene crosses the ambient atmosphere, then the camera lenses before reaching the infrared sensor (in most cases, the sensor uses InSb or HgCdTe technology). The infrared radiation modifies the electrical state of the sensor during the integration time of the image acquisition process. When using infrared focal plane array (IRFPA) cameras, the electrical charges of each sensor (pixel) are then transferred to the acquisition system via a multiplexor before being stored in the computer memory. The quantity to be measured using these systems is rarely infrared radiation but rather temperature. It is thus necessary to convert the digitized radiation into temperature fields of the observed scene as accurately as possible. Each of these steps may generate its own biases, and the combination of these different perturbations will decrease the accuracy and quality of the thermal measurement.

In numerous applications - heat sources or thermophysical property estimation-it is necessary to estimate the spatial and temporal derivatives of the thermal fields obtained by infrared thermography. Consequently, the metrological considerations related to temporal reliability, spatial resolution and calibration remain fundamental 
whatever the chosen analysis (nodal, modal, impulse, modulation, etc.). For instance, the accuracy of the gradient computation is directly linked to the accuracy of the thermal calibration and to the spatial resolution of the thermal measurement, while the accuracy of the time-derivative stems from the calibration procedure and the stability of the time-base. In most cases, these three aspects are combined when dealing with thermophysical property identification, except for a few particular cases such as the thermal diffusivity determination, which 'only' requires precise knowledge of the spatial and temporal parameters.

In this section, the basic laws of thermal radiation are briefly reviewed. They enable the description of the radiation path from the observed object to the camera [GAU 99, PAJ 89] and [PAP 07]. The main calibration methods used for IRFPA are then briefly presented, followed by several metrological considerations regarding infrared measurements. The purely geometrical effects linked to image formation (distortions, aberrations, etc.) are not within the scope of this section, and they will not be discussed hereafter.

\subsubsection{Physics of radiation preliminaries}

The luminance $L$ (unit: W. $\mathrm{m}^{-2} \cdot \mathrm{sr}^{-1}$ ) is the power emitted $(\mathrm{d} \Phi)$ per unit of solid angle $(\mathrm{d} \Omega)$ and per unit of apparent surface $(\mathrm{d} S \cos \theta)$ of an extended source in a given direction defined by the angles $(\theta, \varphi)$ :

$$
L=\frac{d^{2} \Phi}{d \Omega d S \cos \theta} .
$$

The emittance $M$ (unit: W. $\mathrm{m}^{-2}$ ), is the power emitted $\mathrm{d} \Phi$ by a unit surface of an extended source emitting in a whole hemisphere. Consequently, it corresponds to the luminance integrated over all directions of a hemisphere. If the luminance is constant in all directions $(\theta, \varphi)$, it follows Lambert's law and may be formulated as: $M=\pi L$. Monochromatic luminance $L_{\lambda}$ and emittance $M_{\lambda}$ are defined as the luminance and emittance derivatives with respect to the wavelength $\lambda$. When incident radiation encounters an object, a fraction of this radiation is reflected $\Phi_{r}$, another fraction is absorbed by the object $\Phi_{a}$ and the rest is transmitted $\Phi_{t}$. The following factors are then introduced: reflection $\rho_{r}(\lambda, T, \theta, \varphi)$, absorption $\alpha_{r}(\lambda, T, \theta, \varphi)$ and transmission $\tau_{r}(\lambda, T, \theta, \varphi)$. These factors depend on the wavelength $\lambda$, the object temperature $T$ and the radiation direction $(\theta, \varphi)$. Energy conservation implies that the sum of these three factors must always be equal to unity. When the object is at thermodynamic equilibrium, it re-emits the absorbed radiation such that the emissivity factor $\varepsilon_{r}$ is equal to $\alpha_{r}$.

Infrared sensors are sensitive to infrared radiation from an object. Black bodies have the ability to re-emit all received radiation whatever the wavelength (i.e. $\alpha_{r}=\varepsilon_{r}=1$ ). In this case, according to Planck's law, the spectral luminance of an observed black 
10 IR thermographic imaging

body $L_{\lambda}^{\mathrm{CN}}$ can be associated with its temperature $T$ :

$$
L_{\lambda}^{\mathrm{CN}}=\frac{c_{1}}{\pi \lambda 5\left(e^{\frac{c_{2}}{\lambda T}}-1\right)},
$$

where $c_{1}=3.741832 .10^{-16} \mathrm{~W} \cdot \mathrm{m}^{2}$ and $c_{2}=1.438786 .10^{-2}$ K.m. Stefan's law: $L^{\mathrm{CN}}=\pi^{-1} \sigma_{b} T^{4}$ is obtained by integrating Planck's law over the entire wavelength spectrum. Assuming lambertian behavior of the black body, Stefan's law can also be written: $M_{\mathrm{CN}}=\sigma_{b} T^{4}$, with $\sigma_{b}=5.67032 .10^{8} \mathrm{~W} \cdot \mathrm{m}^{-2} \cdot \mathrm{K}^{-4}$ being Stefan-Boltzmann's constant. These two latter expressions allow the radiated flux, measured by an infrared sensor, to be associated with the temperature of the observed black body. In practice, only a few objects behave like black bodies, and the relation between radiation and temperature is established by integrating the monochromatic luminance $L_{\lambda}$ of the observed body on the wavelength spectrum with $L_{\lambda}=\varepsilon_{r}(\lambda, T, \theta, \varphi) L_{\lambda}^{C N}$. Several sets of bodies can be defined according to the specific values taken by parameters $\alpha_{r}, \rho_{r}$ and $\tau_{r}$ : opaque bodies $\left(\tau_{r} \approx 0\right)$, brilliant bodies ( $\rho_{r} \gg 0$ and $\left.\varepsilon_{r} \approx 0\right)$, and gray bodies $\left(\varepsilon_{r} \approx \varepsilon_{\mathrm{r} 0}=\right.$ constant $)$. Measurements are often performed on plane samples, coated with matt black paint in order to obtain high emissivity gray bodies (i.e. Er0 close to 1), with quite a uniform emissivity distribution, independent of the observation direction. The temperature variation should also remain small enough to neglect the temperature-dependence of the emissivity.

\subsubsection{Calibration}

Infrared cameras often have their own setting and acquisition applications, along with basic data-processing applications (conversion from digitized flux to temperature, average and standard deviation computations, display, etc.). Calibration laws proposed by manufacturers are based on the assumption that the sensor's response is linear in terms of emitted flux vs. digitized flux over the whole range between the lower and upper saturation levels of the sensor (i.e. the pixel). The differences between the pixels' responses are interpreted as distributions of gains and offsets to apply to the mean response of the sensor matrix. This operation is often referred to as Non-Uniformity Correction (NUC). The gains and offsets of each pixel are computed so as to obtain uniform distributions of digitized fluxes for two specific images of two uniform thermal scenes taken at different temperatures. To that end, it is advised to use a precision extended black body. Recently, some manufacturers have proposed to relate gain and offset values to the "internal temperature" of the camera in order to account for thermal drifts associated with heat losses that occur between the camera and its environment (Extended NonUniformity Correction: ENUC). The temporal drift of infrared cameras will be addressed more thoroughly in paragraph 16.3.4.

Once the response of each pixel is brought back to the mean response of the sensor 
matrix, a global calibration law is used to relate the average digitized flux to the average temperature. In most cases, the calibration law is taken in the form of a 2- or 3-degree polynomial.

Sensor matrixes always include a certain number of defective pixels (in most cases, less than $0.5 \%$ ). For instance, they correspond to saturated pixels or to "dead" pixels. They are localized using criteria dealing mainly with the discrepancy with respect to the mean response (in terms of digitized flux, gain, offset, etc.). Manufacturers propose to replace the digitized flux value of these pixels by that of their nearest non-defective neighbor (Bad Pixel Replacement, or BPR, operation).

The validity of the "factory" calibration can easily be verified by observing a given thermal scene with a single camera, but using different calibration laws associated with different acquisition settings (integration time, or measurement range). Figure 16-2 illustrates different observations on a black body using different IRFPA cameras. They show that it is appropriate to use the center of the matrix and the middle of the calibration range when using the manufacturer's calibration laws. Furthermore, if the specifications concerning measurement accuracy are more stringent than one Kelvin, it is advisable to perform a complete customized calibration of the sensor matrix with testing conditions and camera configuration (integration time, windowing, etc.) similar to those used for the application.

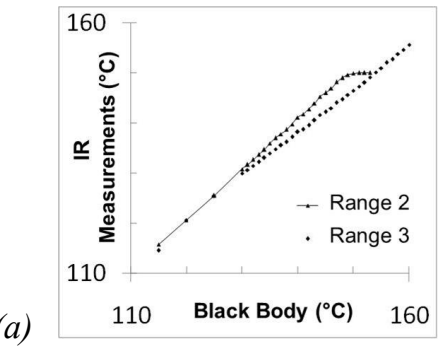

Figure 16.2. Verification of factory calibration using an extended black body: (a) Comparison between two ranges of a single camera (FLIR SC1000), (b) Comparison between several pixel responses (CEDIP IRC 320-4LW)

This calibration overcomes the limitations inherent to the $N U C-B P R$ procedures (linearity assumption valid far enough from saturation for the $N U C$, introduction of 
a strong spatial correlation between neighboring pixels for the $B P R$ operation). However, this calibration requires an extended black body in order to have a uniform radiation source at different temperature levels. The calibration law of each pixel can be chosen as a polynomial or as a Planck-like function. The calibration coefficients are obtained by approximating, generally in the least squares sense, the couples (digitized radiation-temperature) by the chosen calibration function.

Defective pixels are then localized using a criterion for measuring the mismatch between the calibrated and specified temperature. The BPR operation is not performed: temperatures of the defective pixels are not taken into account in the subsequent data-processing. A specific pixel-to-pixel calibration is detailed in [HoN 05].

\subsubsection{Temporal analysis}

The integration time sets the duration during which radiation resulting from the thermal scene will be collected by the camera. For this reason, it determines the temporal resolution of the device. As the image transfer time to the storage memory is often much higher than the integration time (a few ms vs. some $\mu \mathrm{s}$ ), the camera thus does not see the scene most of the time, which is particularly penalizing for observing fast phenomena.

Once the integration time is chosen, the temporal analysis can be disturbed by the absence of some images in the stored sequence. Depending on the equipment, a temporal shift of one or two images can occur at the beginning of the sequence. This is due to the fact that the first stored image corresponds to the one that was captured when the starting order occurred, not the actual image at the beginning of the sequence. A simple sequence shift is enough to correct this edge effect.

The second, more penalizing, problem is the absence of some images in a film. This problem is relatively unimportant in terms of visualization, but can become critical in image processing when time is involved. Algorithms that are compatible with variable acquisition frequencies are then required. To count and isolate times from the missing images, it is possible to directly read time information in the files from the camera, provided that they have been accurately stored, i.e. sufficient with respect to the acquisition frequencies used. Depending on the camera model, the number of missing images can thus range from one to several dozens. 

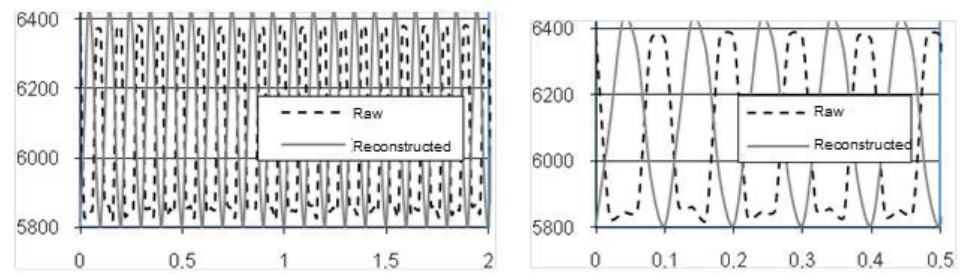

Figure 16.3: Errors induced by the loss of images: in lock-in thermography, the amplitude is not really affected (left) but the phase is strongly distorted (right)

Figure 16.3 presents the artifacts observed in the case of a numerical lock-in procedure applied to a series of 500 images in which only two images are missing. If the amplitude is not very affected, the phase has a completely erratic behavior, and takes a value that depends directly on the number and the phase of the missing images.

\subsubsection{Spatial resolution}

The adoption of focal plane array technology has led to clear improvements in image quality (Figure 16-4).

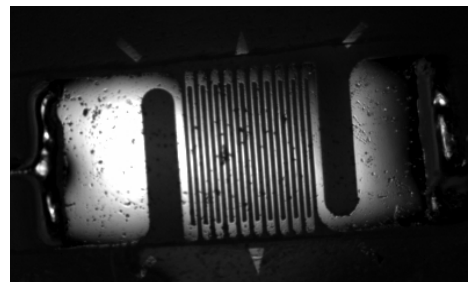

Figure 16.4: Extensometric gauge (tracks of approximately $20 \mu \mathrm{m}$ ) observed with an M1 lens.

However, the independence of each sensor relative to its neighbors must be checked. One of the most current tests for characterizing such equipment is the Slit Response Function (SRF) test: the camera focuses on a thermal slit (side-cooled slit of variable width placed in front of a hot plate), and the following function is studied (contrast): 
14 IR thermographic imaging

$$
F R F=\frac{V(x)-V_{\text {min }}}{V_{\text {max }}-V_{\text {min }}},
$$

where $V(x)$ is the value recorded for a slit width equal to $x, \mathrm{~V}_{\max }$ is the value recorded when the slit is wide open $(\mathrm{x} \rightarrow \infty)$ and $\mathrm{V}_{\min }$ is the recorded value on the cooled part (Figure 16-5a). In general, it is assumed that, for $320 \times 240$ pixel cameras, to obtain a good measurement the object must be projected on at least two detectors. Thus, with a lens magnification of 1 (M1) and a matrix periodicity of 30 $\mu \mathrm{m}$, truly independent information can only be obtained at each step of $60 \mu \mathrm{m}$.

A study of this SRF for different positions clearly shows (Figure 16-5b) that the pixels are definitely more correlated on the edges of the image than in the center. Note that there is indeed a problem of correlation between close measurement points, i.e. on the one hand, only the contrast (and by no means the average value) is affected and, on the other hand, there is convolution of the thermal scene by this response function. Consequently, a simple geometrical correction (e.g. of repositioning of the points in the image, or amplification and/or offsets applied to each pixel) is necessary to recover the real quantitative image of the scene, in addition to a de-convolution procedure.

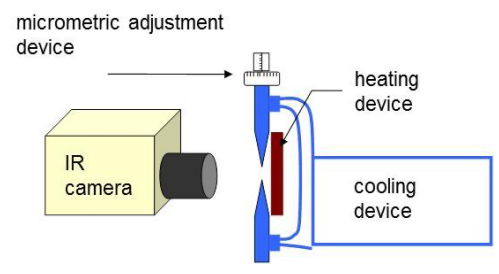

(a)

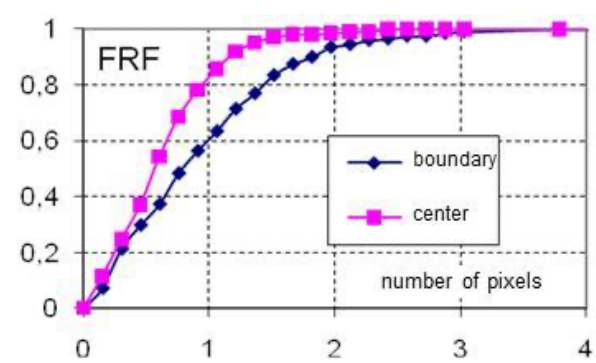

(b)

Figure 16.5 : (a) Slit Response Function; (b) SRF near the edge of the array compared to SRF at the center (CEDIP IRC 320-4 LW camera)

\subsubsection{Thermal noise and thermal drift}

\subsubsection{Thermal stability of devices}

In order to reduce radiation in the vicinity of the infrared sensors, the array is cooled at approximately $80 \mathrm{~K}$. In new-generation IR cameras, cooling is done using a Stirling cycle engine, which has replaced the liquid nitrogen cooling systems of 
older cameras. However, cameras have gained in portability, but with a loss of performance: the cooling which was quasi-instantaneous with nitrogen now requires at least 10 min before any measurement is possible (Figure 16.6a).
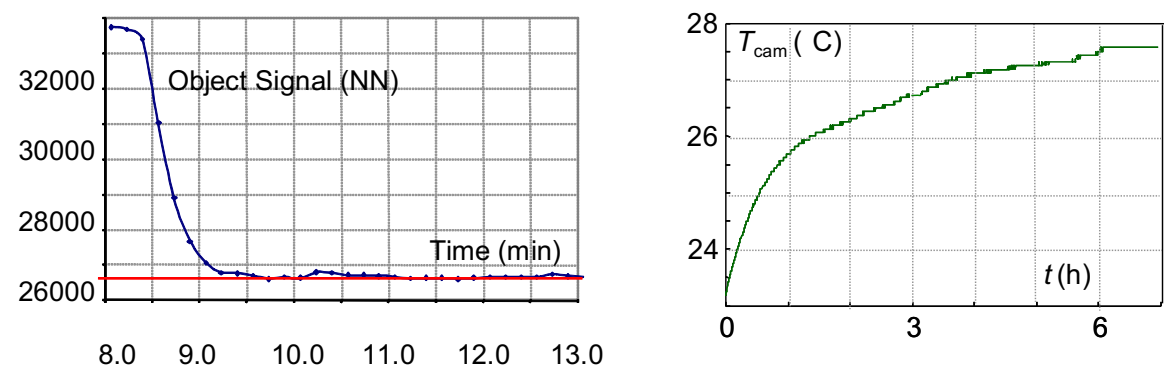

Figure 16.6 : (a) Cooling CEDIP IRC320-4LW, (b) thermal drift CEDIP JADE III

In addition, once cooling is achieved, a slow drift of about 1 to $5 \mathrm{mK}$ per second can occur with certain equipment, sometimes over durations reaching a few hours (Figure 16.6b). Since these internal temperature drifts in the camera modify the sensor responses, it is appropriate in many situations to wait until the camera temperature is stabilized, or to take this internal drift into account in the conversion of the digitized signal into temperature (compensated NUC). In addition, certain lower-quality devices have instabilities of 0.5 or even $1 \mathrm{~K}$, which is incompatible with quantitative measurements.

\subsubsection{Environmental stability}

The signal measured by a camera (which is roughly an intensity) comes primarily from the object (which is assumed to be opaque and gray in the camera's spectral range), but also, to a lesser extent, from the environment and atmosphere (Figure 167). If the environment can be considered as an integral radiator of temperature $T_{e n v}$ and if the atmosphere between the target and the camera is isothermal at temperature $T_{\text {atm }}$, considering a coefficient of transmission $\tau_{\text {atm }}$, the measured intensity $L_{m e s}$ can be formulated as a function of the intensity of a black body at the object temperature $\mathrm{L}^{0}$ :

$$
L_{\text {mes }}=\tau_{\mathrm{a}} \cdot \varepsilon \cdot L^{0}\left(T_{\mathrm{obj}}\right)+\tau_{\mathrm{a}}(1-\varepsilon) L^{0}\left(T_{\mathrm{env}}\right)+\left(1-\tau_{\mathrm{a}}\right) L^{0}\left(T_{\mathrm{atm}}\right)
$$

For short distance measurements (about a few dozens of $\mathrm{cm}$ ), the atmosphere can 
16 IR thermographic imaging

reasonably be considered as being transparent, and thus:

$$
L_{\text {mes }}=\varepsilon L^{0}\left(T_{\text {obj }}\right)+(1-\varepsilon) L^{0}\left(T_{\text {env }}\right)
$$

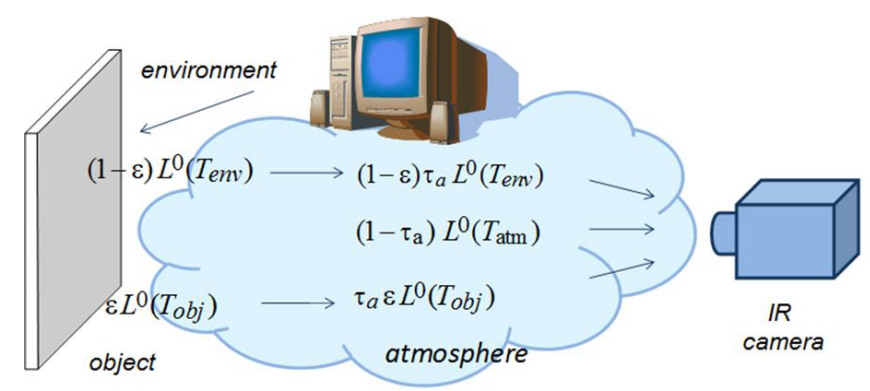

Figure 16.7: Simplified radiometric balance

This equation shows that the environment must be reasonably well controlled in order to limit the influence of parasitic radiation (reflection from a radiator or any other radiative IR source, or even from the operator!). This precaution is all the more important when the measured temperature increases are minor. In addition, using a high emissivity coating (thus of low reflectivity) is obviously advantageous to minimize the parasitic flow/object flow ratio.

Along the same lines, note also the presence of the Narcissus effect (reflection of the cold detector on the scene), which is often observed when using a macro lens (e.g. lens magnification of 1 , [PRON, 00]). Usually, this is only an offset map which is superimposed on the scene, and which can thus be offset by subtraction of a reference image.

Last but not least, possible environmental instabilities could modify the exchange conditions between the sample and its environment and thus must be taken into account, especially when there are marked temperature variations over time. Consequently, it is useful to check that room temperature variations at the different hours of the day (Figure 16.8) do not significantly influence the measurements. 


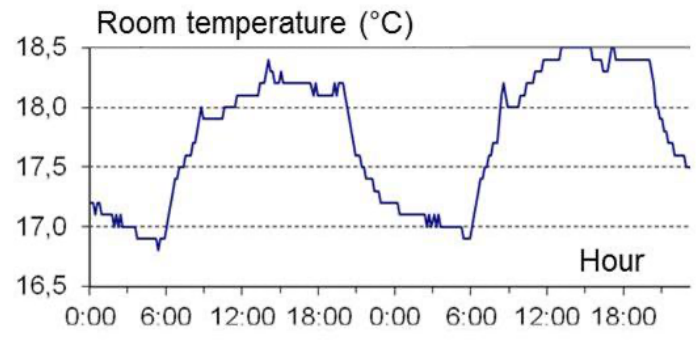

Figure 16.8: Environmental temperature variations over $24 \mathrm{~h}$

\subsection{Heat diffusion models and identification methods}

An ultimate goal of thermal image processing could be the determination of source fields at any point in a 3D structure while taking variations in thermophysical properties of the material into account throughout the process. However, this is (currently) out of reach for metrology reasons related to the physics of radiation and IR techniques, but also for mathematical reasons (surface measurements, inverse illposed problem). The models proposed hereafter will focus on diffusion problems in simplified systems, either 2D (thin plate, semi-infinite medium) or 1D (straight beam) and even 0D (material volume placed within a homogeneous medium uniformly cooled or heated). We will however introduce, through integral transforms, direct relationships between full-field temperature measurements and certain types of sources in the case of thick 3D media. The following section puts forward equations that enable us to estimate thermophysical parameters or heat sources using thermographic images. The many possibilities offered by integral transformations of temperature fields, in relation to the heat transfer model, are the cause of numerous infrared image issues. In what follows, we will refer to operational application examples to highlight this.

\subsubsection{Diffusion equation for thin plates}

The medium in question is a thin plate of length $L$, width $l$ and thickness $e$. The corresponding coordinates are $(x, y, z)$, respectively. Within the framework of our experimental tests, the following hypotheses are often accepted:

- the specific heat and conduction coefficient are constant material characteristics, independent of the internal state,

- as the tests are quasi-static, the convective terms are neglected in the particular time derivative. Note that the combination of IR and digital image correlation (DIC) techniques enable us to monitor the temperature of material surface elements (SEM) and to consider the convected heat [BER 08, Chr 09, Chr 10]. 
In some cases (e.g. necking lips of polymers [MUR 08]), the convected heat may reach up to 40 percent of the heat source.

To reduce it to a $2 \mathrm{D}$ problem, the idea here is to consider that the averaged value of the source $s(x, y, z, t)$ over the thickness is sufficiently representative of this distribution throughout this thickness. We classically define:

$$
\bar{f}(x, y, t)=\frac{1}{e} \int_{-\frac{e}{2}}^{+\frac{e}{2}} f(x, y, z, t) \mathrm{d} z
$$

as the averaging operation. Assuming constant thermophysical parameters and applying the averaging operation to the heat diffusion equation [16.10], we obtain:

$$
\rho C\left(\frac{\partial \bar{\theta}}{\partial t}+\frac{\bar{\theta}}{\tau_{\mathrm{th}}^{2 \mathrm{D}}}\right)-k\left(\frac{\partial^{2} \bar{\theta}}{\partial x^{2}}+\frac{\partial^{2} \bar{\theta}}{\partial y^{2}}\right)=\bar{s} \text { with } \rho C \frac{\bar{\theta}}{\tau_{\mathrm{th}}^{2 \mathrm{D}}} \approx-\frac{k}{e}\left[\frac{\partial \theta}{\partial z}\right]_{-\frac{e}{2}}^{+\frac{e}{2}},
$$

where out-of-plane heat losses are estimated under the assumption of linear heat fluxes (Fourier boundary conditions) and where the time constant $\tau_{\text {th }}^{2 \mathrm{D}}$ characterizes the heat fluxes through the surfaces defined by $z= \pm \frac{e}{2}$.

\subsubsection{Diffusion equation for straight beams}

For some sample shapes, it may be interesting to work only with temperature and source profiles along a straight beam. This represents a gain with respect to data storage, processing speed and the quality of the signal-to-noise ratio of thermal data. This approximation is all the more acceptable since the heat source distribution slightly fluctuates within a cross-section $S$, inducing a representative average value. Assuming that thermophysical parameters remain constant over each cross-section and then integrating equation [16.10] over S, we obtain a $1 \mathrm{D}$ version of the heat diffusion equation. Noting:

$$
\bar{f}(x, t)=\frac{1}{S} \int_{-\frac{l}{2}}^{+\frac{l}{2}} \int_{-\frac{e}{2}}^{+\frac{e}{2}} f(x, y, z, t) \mathrm{d} z \mathrm{~d} y,
$$

the diffusion equation becomes:

$$
\rho C\left(\frac{\partial \bar{\theta}}{\partial t}+\frac{\overline{\bar{\theta}}}{\tau_{\mathrm{th}}^{1 \mathrm{D}}}\right)-k\left(\frac{\partial^{2} \bar{\theta}}{\partial x^{2}}\right)=\bar{s} \text { with } \frac{\overline{\bar{\theta}}}{\tau_{\mathrm{th}}^{1 \mathrm{D}}} \approx-\frac{k}{\rho C}\left(\overline{\overline{\frac{\partial^{2} \theta}{\partial y^{2}}+\frac{\partial^{2} \theta}{\partial z^{2}}}}\right) .
$$

The approximation of lateral heat losses is still based on the assumption of linear heat exchange temperature at the boundary $\partial S$ of each cross-section $S$. The heat exchange coefficient $h$ between the specimen and the surrounding air is assumed to be constant: 
IR thermographic imaging 19

$$
-k \operatorname{grad} \theta \cdot n=h \theta \text {, }
$$

where $n$ is the outward-pointing normal vector to $\partial S$.

Application examples on the necking of steel and polymers have been presented in [CHR 00] and [MUR 08].

\subsubsection{Diffusion equation for a monotherm material volume element}

In the classic framework of tensile loading on homogeneous plates, which is of practical importance, we can reduce the heat diffusion problem in the center of the sample gauge part to an ordinary differential problem with respect to time. For this, the material must have constant thermophysical characteristics with a uniform load, thus resulting in a uniform heat source distribution. Moreover, the initial temperature field is uniform, and finally the thermal boundary conditions are linear (Fourier condition) and symmetrical on each pair of facing surfaces. Under these conditions, it can be shown [CHR 00] that the solution to the 3D diffusion problem is almost completely borne by the first eigen-function of the spectral solution. The $3 \mathrm{D}$ diffusion problem reduces to the following differential equation:

$$
\rho C\left(\frac{\mathrm{d} \theta}{\mathrm{d} t}+\frac{\theta}{\tau_{\mathrm{eq}}}\right) \approx s,
$$

where $\theta$ is the temperature variation recorded in the center of the specimen gauge part, and with the time constant $\tau_{\text {eq }}$ characterizing the local heat losses [CHR 95]. This simple heat equation of ODE form has been used to study the behavior of materials subjected to high cycle fatigue tests [BOU 04, DOU 09].

\subsubsection{Integral transforms and quadrupole method related to thick media}

A direct analytical relation between the observable temperature field (from an IR camera) and the heating source field is convenient for implementing estimation methods related to thick media. The characterization of thermophysical properties requires calibrated heat excitation, often imposed at the sample surface. Instead of thermophysical properties, when the characterization of an unknown internal heating source field is investigated, the transient point-source response (green function) can be conveniently considered.

The main idea is to consider the general heat equation reduced to a case depending only on the space variable $x$ orthogonal to the observed sample surface. A 1D heat transfer versus the $x$-direction is then considered, when the heat losses are assumed to be negligible $\left(\tau_{\mathrm{th}}^{1 \mathrm{D}} \rightarrow+\infty\right)$. An expression very similar to [16.23] is then obtained. 
20 IR thermographic imaging

Then, based on the Laplace transformation, suitable direct expressions of the temperature response to localized heating may be formulated. The general $1 \mathrm{D}$ heat equation related to a homogeneous wall of thickness $e$ is then:

$$
\frac{\partial^{2} \theta}{\partial x^{2}}=\frac{1}{a} \frac{\partial \theta}{\partial t}
$$

where $a=k / \rho C$ is the thermal diffusivity of an homogeneous medium. This thermophysical parameter (in $\mathrm{m}^{2} \mathrm{~s}^{-1}$ ) is crucial for estimating the spatial distribution of source terms. The previous expression is obtained with a Laplace transform such that:

$$
\tilde{\theta}(x, p)=\int_{0}^{\infty} \exp (-p t) \theta(x, t) \mathrm{d} t,
$$

the Laplace variable $p$ can be related to a frequency in $s^{-1}$. If $\theta(x, t=0)=0$ whatever $x$, the Laplace transform of expression [16.26] then yields:

$$
\frac{\mathrm{d}^{2} \tilde{\theta}}{\mathrm{d} x^{2}}=\frac{p}{a} \tilde{\theta}
$$

The definition of the Laplace transform of the heat flux at a given depth $x$ is then:

$$
\tilde{q}(x, p)=-k \frac{d \tilde{\theta}}{d x}(x, p) \text {. }
$$

The following ordinary differential system expressed with the [temperature, flux] vectors in the Laplace space yields:

$$
\frac{d}{d x}\left[\begin{array}{l}
\tilde{\theta} \\
\tilde{q}
\end{array}\right]=\left[\begin{array}{cc}
0 & -1 / k \\
-\rho C p & 0
\end{array}\right]\left[\begin{array}{l}
\tilde{\theta} \\
\tilde{q}
\end{array}\right],
$$

Integration on domain $[0, e]$ gives the solution of system [16.30], such that:

$$
\left[\begin{array}{l}
\tilde{\theta}(x=0, p) \\
\tilde{q}(x=0, p)
\end{array}\right]=\left[\begin{array}{ll}
A & B \\
C & D
\end{array}\right]\left[\begin{array}{l}
\tilde{\theta}(x=e, p) \\
\tilde{q}(x=e, p)
\end{array}\right],
$$

where $r_{a}(p)=\sqrt{\frac{p}{a}}$ :

$$
A=D=\cosh \left(r_{a} e\right), B=\frac{\sinh \left(r_{a} e\right)}{k r_{a}} \text { and } C=k r_{a} \sinh \left(r_{a} e\right)
$$


This kind of approach was previously used for the analogical description of electrical circuits called quadrupoles. This name is related to such approaches, even involving heat transfer [CAR 59, MAI 00].

In the case of pulse heating at $x=0\left(\widetilde{q}(x=0, p)=Q\right.$, with $Q$ in $\left.\mathrm{J}_{\mathrm{m}} \mathrm{m}^{-2}\right)$ for a given sample assumed to be adiabatic, it gives:

$$
\left[\begin{array}{c}
\tilde{\theta}(x=0, p) \\
Q
\end{array}\right]=\left[\begin{array}{ll}
A & B \\
C & D
\end{array}\right]\left[\begin{array}{c}
\tilde{\theta}(x=e, p) \\
0
\end{array}\right] .
$$

The Laplace transform of the temperature response, measured by the infrared camera on the front face $(x=0)$ gives:

$$
\tilde{\theta}(x=0, p)=Q \frac{A}{C}=Q \frac{\cosh \left(r_{a} e\right)}{k r_{a} \sinh \left(r_{a} e\right)},
$$

and on the rear face $(x=e)$ :

$$
\tilde{\theta}(x=e, p)=\frac{Q}{C}=\frac{Q}{k r_{a} \sinh \left(r_{a} e\right)},
$$

A simple expression between the front face and the rear face temperatures, at short time or high frequency (large $p$ values) can be deduced such that:

$$
\tilde{\theta}(x=e, p)=\tilde{\theta}(x=0, p) \cdot \exp \left(-r_{a} e\right)
$$

In addition to having the advantage of reflecting explicit relationships in a Laplace space, expressions [16.34] to [16.36] can be tailored to problems with lateral heat losses and/or in 2D or 3D situations. This analytical method allows us to consider very thick layers (semi-infinite media, see section 16.4.4.2) or very thin layers (see section 16.4.4.3) with asymptotic expansions. Transient periodic phenomena are also conveniently considered by similar expressions when the Laplace transform is replaced by a Fourier transform. The main advantage is that heat transfer can be represented through multilayered samples by a simple matrix product (see [BAT 94, MAI 00]).

Such expressions and the resulting asymptotic expansions are also the starting 
point for designing instrumental methods for measuring thermophysical properties (diffusivity, or thermal contact resistances). 1D analytical considerations have provided the basis for flash methods (see [HAY 04] for a review of these methods).

\subsubsection{Multidimensional cases}

$1 \mathrm{D}$ cases are often inadequate when the front face heat flux (in $x=0$ ) is spatially non-uniform, for instance when heating is obtained with a laser spot. A 2D or 3D transient transfer assumption must then be considered, even with a non-isotropic sample. The general equation in the principal axes of anisotropy is then:

$$
\frac{\partial 2 \theta}{\partial x^{2}}+\frac{k_{y}}{k_{x}} \frac{\partial 2 \theta}{\partial y^{2}}+\frac{k_{z}}{k_{x}} \frac{\partial 2 \theta}{\partial z^{2}}=\frac{1}{a_{x}} \frac{\partial \theta}{\partial t},
$$

where $k_{x}, k_{y}, k_{z}$ are the thermal conductivities following the principal directions $x, y$, $\mathrm{z}$ with $a_{x}=k_{x} / \rho C$ : thermal diffusivity related to direction $x$.

A simple situation can be reduced to a sample which is adiabatic on the lateral faces (in $y=0$ et $y=L$ and in $z=0$ and in $z=l$ ) :

$$
\left.\frac{\partial \theta}{\partial y}\right|_{y=0}=\left.\frac{\partial \theta}{\partial y}\right|_{y=L}=\left.\frac{\partial \theta}{\partial z}\right|_{z=0}=\left.\frac{\partial \theta}{\partial z}\right|_{z=l}=0,
$$

A Fourier integral transform related to the space variables $y$ and $z$ and a Laplace transform related to the time variable $t$ allow us to obtain a formal expression similar to the previous $1 \mathrm{D}$ case. Here the adapted Fourier-Laplace transform is as follows:

$$
\theta\left(x, \alpha_{n}, \beta_{m}, p\right)=\int_{0}^{L} \int_{0}^{l} \int_{0}^{\infty} \theta(x, y, z, t) \cos \left(\alpha_{n} y\right) \cos \left(\beta_{m} z\right) \exp (-p t) \mathrm{d} x \mathrm{~d} y \mathrm{~d} t
$$

where pulsations $\alpha_{n}$ and $\beta_{m}$ are defined by $\alpha_{n}=\frac{n \pi}{L}$ and $\beta_{m}=\frac{m \pi}{l}$.

The general equation in the transformed space, where $\theta(x, y, z, t=0)=0$, is then

$$
\frac{d^{2} \tilde{\theta}}{d x^{2}}-\frac{k_{y}}{k_{x}} \alpha_{n}^{2} \tilde{\theta}-\frac{k_{z}}{k_{x}} \beta_{m}^{2} \tilde{\theta}=\frac{p}{a_{x}} \tilde{\theta},
$$

The method described previously in the $1 \mathrm{D}$ case can be extended with the same 
formal expressions, by then considering: $r_{a}^{2}=\frac{p}{a_{x}}+\frac{k_{y}}{k_{x}} \alpha_{n}^{2}+\frac{k_{z}}{k_{x}} \beta_{m}^{2}$.

The simultaneous estimation of parameters: $a_{x}, k_{y} / k_{x}$ and $k_{z} / k_{x}$ is then obtained by considering the marginal averaging of temperature images related to zero pulsations $\left(\alpha_{\mathrm{n}}=0, \beta_{\mathrm{n}}=0\right)$. These expressions enable the modeling of many experimental situations related to the use of infrared thermography devices. Simultaneous estimations of thermal diffusivity tensor terms are available, for instance in [PHI 95, KRA 04].

\subsubsection{Semi-infinite medium}

$3 \mathrm{D}$ transient cases in semi-infinite media are easily deduced from the previous expressions. At the front face (at $x=0$ ), point source pulse heating at $y=z=0$ is expressed by a Laplace-Fourier transformation by: $Q_{0}$ (in J) which is a real constant in Laplace-Fourier space. This kind of heating can nowadays be conveniently implemented with a focused laser spot (at $y=z=0$ ) in pulsed mode. The temperature response, when $e$ tends to infinity in expression [16.34] extended to the 3D case (Laplace-Fourier transform), yields:

$$
\tilde{\theta}\left(x, \alpha_{n}, \beta_{m}, p\right)=\frac{Q_{0}}{k_{x} r_{a}} \exp \left(-r_{a} x\right) \text { and } \tilde{\theta}\left(x=0, \alpha_{n}, \beta_{m}, p\right)=\frac{Q_{0}}{k_{x} r_{a}}
$$

This expression is very important in order to assess temperature behaviour at the surface $($ at $x=0)$ of any thick medium at short times.

If the inverse expression of the heat flux at $x=0$ is considered versus the observed temperature at $x=e$, it then yields:

$$
Q_{0}=\widetilde{\theta}\left(x=e, \alpha_{n}, \beta_{m}, p\right) k_{x} r_{a} \exp \left(+r_{a} e\right) .
$$

This expression is not stable and is very sensitive to measurement noise when directly applied to experimental temperature observations. It amplifies the high frequency measurement noise exponentially even if this noise amplitude is lower than the signal, especially when the observation depth $e$ is large. This example illustrates the ill-posed nature of the attempt to estimate the characteristics of an indepth heating source (depth, size, form, intensity, etc.) from surface temperature observations and analysis (see for example [BEC 77]).

Expression [16.41] can also be considered in time-space by a Laplace inverse transform of the previous expression and remaining the Fourier transform. 
24 IR thermographic imaging

$$
\tau\left(x, \alpha_{n}, \beta_{m}, t\right)=\left(Q_{0} / \rho c\right)\left(e^{\left(-x^{2} /\left(4 a_{x} t\right)\right)} / \sqrt{\pi a_{x} t}\right) e^{\left(-a_{y} \alpha_{n}{ }^{2} t\right)} e^{\left(-a_{z} \beta_{m}{ }^{2} t\right)}
$$

A suitable approximation of the inverse Fourier transform can then be considered when $l$ and $L$ are large:

$$
\theta(x, y, z, t)=\left(Q_{0} / \rho C\right)\left(\mathrm{e}^{\left(-x^{2} /\left(4 a_{\mathrm{x}} t\right)\right)} / \sqrt{\pi a_{x} t}\right)\left(\mathrm{e}^{\left(-y^{2} /\left(4 a_{\mathrm{y}} t\right)\right)} / \sqrt{\pi a_{\mathrm{y}} t}\right)\left(\mathrm{e}^{\left(-z^{2} /\left(4 a_{\mathrm{z}} t\right)\right)} / \sqrt{\pi a_{\mathrm{z}} t}\right)
$$

This expression is then considered to be separable or presented as a product of three terms depending on the $x, y$ and $z$ directions.

Expression [16.44] then contains the main characteristics of the $3 \mathrm{D}$ diffusive heat transfer.

If the observed temperature field is summed versus the $y$ and $z$ direction (or if both $\alpha_{n}=0$ and $\beta_{m}=0$ are considered in expression [16.43]), a 1D expression related to a purely diffusive transfer versus the $x$ direction and the parameter to be estimated is the thermal effusivity related to the $x$ direction: $\sqrt{k_{x} \rho C}$ :

$$
\int_{0}^{L} \int_{0}^{l} \theta(x=0, y, z, t) \mathrm{d} y \mathrm{dz}=\frac{Q_{0}}{\sqrt{k_{x} \rho C} \sqrt{\pi t}}
$$

This result provides the basis of the hot plane thermal effusivity estimation method. Uniform heating is applied (generally with the contact of a uniformly deposited heating resistor network) on the front face of the sample and the transient front face temperature response is then analyzed (see [GUS 91] and [FUD 05]). Generally, the inertia of the heating device and temperature probe must be taken into account with this method. Instead of a temperature probe, an infrared camera may be used to conveniently consider not only one sensor (or one image pixel) in the case of uniform heating at $x=0$, but the whole sensor field, which may be averaged (simultaneously averaging all pixels of the image) in the case of point source heating. If heating is generated by a laser spot, the inertia of this heating and of the temperature sensor is avoided, and such optical methods may be used for investigations related to very short times or high frequencies.

If the medium is heated with a line source in the $y$ direction (instead of a point source), this is equivalent to the consideration of averaged pixel signals versus $y$ (or $\left.\alpha_{n}=0\right)$ in the case of a point source. At $z=0$, the expression is then: 


$$
\int_{0}^{L} \theta(x=0, y, z=0, t) \mathrm{d} y=\frac{Q_{0}}{\sqrt{k_{x} \rho C} \sqrt{\pi t}} \frac{1}{\sqrt{a_{z}} \sqrt{\pi t}}=\frac{Q_{0}}{\sqrt{k_{x} k_{z}} \pi t}
$$

The analysis of the temperature response to the line source is also called the hot wire method. This method has led to many experimental applications and variants with solid sensors (platinum wire for both heating and temperature measurement, thermocouple-wire systems, etc., see [CAR 59, FUD 05]). Contrary to the hot plane method, this method is sensitive to thermal conductivity rather than thermal effusivity. In the case of orthotropic media, the estimated thermal conductivity is related to the harmonic averaging of the two-directional transfer, then the estimated thermal conductivity is $\sqrt{k_{x} k_{z}}$. The development of infrared cameras has enabled the simultaneous implementation of hot plane, hot wire and point source methods by a simple directional averaging of the temperature response mapped with a single point heating source. The prime advantage with a laser spot and an infrared camera is to avoid the inertia of solid sensors. The main problem is then to manage the radiative heating of the sample. Even if this condition is not fulfilled, IR camera measurement is useful to compare the ratio between the parameters considered here.

\subsubsection{Thin plate}

If the studied medium is very thin ( $e$ small), expression [16.34] can be reduced to a 2D case which justifies the Laplace-Fourier transform of approximation [16.16] presented in section 1.3.1:

$$
\tilde{\theta}\left(x=0, \alpha_{n}, \beta_{m}, p\right)=\frac{Q_{0}}{e\left(\rho C p+k_{y} \alpha_{n}^{2}+k_{z} \beta_{m}^{2}\right)}
$$

Such analytical expressions can be studied and implemented under many different forms and represent the first steps (forward models) for thermal diffusivity estimation (even thermal diffusivity mapping) or for identifying functions related to source terms inside the medium. Such solutions can be considered in real space with inverse transforms, or directly in the transformed space [DEG 96, BAT 01].

\subsubsection{Inverse Laplace Transform- Stehfest Algorithm}

Numerical inversion algorithms for the Laplace transform are very useful if the previous expressions are considered. No universal algorithm exists, but the very convenient Stehfest algorithm is presented here and is suitable for the calculation of temperature responses in the case of pulse heating (see [MAI 00] for further details related to such algorithms).

The method consists of seeking the time-space function $f(t)$ as a linear expression such that: 


$$
f(t)=\frac{\operatorname{Ln}(2)}{t} \sum_{j=0}^{j=N} V_{\mathrm{j}} F\left(\frac{j \operatorname{Ln}(2)}{t}\right)
$$

where $F(p)$ is the Laplace transform of $f(t) . N$ depends on the floating-point precision of the computer. With single precision, $N=10$ is often the most suitable choice, with the following $V_{j}$ coefficients: $V_{1}=1 / 12, V_{2}=-385 / 12, V_{3}=1279, V_{4}=$ $46871 / 3, V_{5}=505465 / 6, V_{6}=-473915 / 2, V_{7}=1127735 / 3, V_{8}=-1020215 / 3, V_{9}=328125 / 2$, $V_{10}=-65625 / 2$.

\subsubsection{Inverse Fourier transform}

A substantial body of scientific literature is related to Fourier transform applications (see for example [PRE 07]). Generally, for heat transfer experiments, classic solutions related to cosine Fourier transforms are adapted to adiabatic boundary conditions at $y=0$ and $y=L$ and at $z=0$ and $z=L$ :

$$
\tilde{\theta}\left(\alpha_{n}\right)=\int_{0}^{L} \theta(y) \cos \left(\alpha_{n} y\right) \mathrm{d} y
$$

The inverse transform is then given by the following expression:

$$
\theta(y)=\frac{1}{L} \hat{\theta}(0)+\frac{2}{L} \sum_{n=1}^{\infty} \cos \left(\alpha_{n} y\right) \tilde{\theta}\left(\alpha_{n}\right), \text { with } \alpha_{n}=\frac{n \pi}{L}
$$

Integral [16.44], applied to discrete infrared camera observations, is approximated by trapezoidal rules [PRE 07]. Usually only a finite number $N$ of pixels provides information about the temperature distribution and then allows access to a finite number $N$ of transformed temperatures $\tilde{\theta}\left(\alpha_{n}\right)$. An extension to 2D transient problems is then possible.

\subsection{Concluding comments and prospects}

In conclusion, research in the mechanics of materials domain concerned by infrared thermography has a broad scope. The thermomechanical behavior of material under mechanical and thermal loading is too often assessed by identifying the material temperature and comparing it to that of the close environment (e.g. room 
temperature). When its role is not reduced to this parameter, temperature is often referred to as a control variable. These approximations surely mask important phenomena that cannot be identified without adopting a true thermomechanical approach to material behavior. Advances in thermographic imaging now enable access to quantitatively reliable temperature measurement fields. These fields can then be used to assess certain physical coefficients, such as the local diffusivity or conduction of the studied material. In the near future, these space distributions of thermophysical properties could be used to detect material irregularities that are natural or induced by mechanical and thermal loadings. Once these thermophysical characteristics are known, temperature fields can then be used to estimate heat sources accompanying the deformation, while reflecting the irreversibility of the deformation mechanisms and/or thermomechanical coupling effects.

The examples mentioned here are limited to quasi-static transformations at constant temperature. Although much remains to be done, it should not be considered that this is the only application area for infrared techniques. Increasingly, thermographic analysis can address the problem of modeling the behavior of materials at high temperatures and dynamics. Indeed, during physical treatments (hardening, annealing, etc.), or welding processes (melting, solidification), thermomechanical coupling phenomena (phase transitions, precipitation kinetics) are of critical importance. The constant development of faster IR cameras already allows mechanical engineers to address the dynamic behavior of materials in which, again, couplings play an important role in the instability propagation mode (adiabatic shear band, auto-catalytic cycle).

\subsection{References}

[BAT 94] Batsale J.C., Maillet D., Degiovanni A., "Extension de la méthode des quadripôles thermiques à l'aide de transformations intégrales." Application au défaut plan bidimensionnel », Int. J. Heat Mass Transfer, 37, (1), pp. 111-127, 1994.

[BAT 01] BatSale J.C. , Le Niliot C., " Estimation de paramètres par thermographie IR ", Métrologie Thermique et Techniques inverses, vol 1, chap.4, Presses Universitaires de Perpignan, pp. 179-236, 2001.

[BAt 04] Batsale J.C., Battaglia J.L., Fudym O., " Autoregressive algorithms and spatially random flash excitation for $3 \mathrm{D}$ non destructive evaluation with infrared cameras ", Int. J. on Quantitative InfraRed Thermography (QIRT), 1, pp. 5-20, 2004.

[BeC 77] BeCK J. V., ARNOLD K-J., " Parameters estimation in engineering and science ", Wiley, NY, 1977.

[Ber 08] Berthel B., Chrysochoos A., Wattrisse B., Galtier A., " Infrared image processing for the calorimetric analysis of fatigue phenomena ", Exp. Mechanics, 48, (1), pp. 79-90, 2008. 
[Bou 04] Boulanger T, Chrysochoos A, Mabru C, " Calorimetric analysis of dissipative and thermoelastic effects associated with the fatigue behavior of steels ", Int. J. of Fatigue, 26-3, pp. 221-229, 2004.

[BRE,82] BRÉMOND P., (1982), " Développement d'une instrumentation infrarouge pour l'étude des structures mécaniques", thèse de doctorat, Université de Marseille, France.

[CAR 59] Carlslaw H.S., Jaeger J.C., " Conduction of Heat in solids ", Oxford, Clarendon Press, 1959.

[CHA 84] CHADWICK P., CREASY C.F.M, " Modified entropic elasticity of rubberlike materials ", J. Mech. Phys. Solids, 32, pp. 337-357, 1984.

[Chr 95] Chrysochoos A., " Analyse du comportement thermomécanique des matériaux par thermographie infrarouge", Actes du colloque Photomécanique 95, Cachan, pp.203-211, 1995.

[Chr 00] Chrysochoos A., Louche H., " An infrared image processing to analyze the calorific effects accompanying strain localization ", Int. J. Eng. Sci., 38, pp. 1759-1788, 2000.

[Chr 09] Chrysochoos A., Wattrisse B., Muracciole J.-M., El KaÏM Y., " Fields of stored energy associated with localized necking of steel ", J. of the Mechanics of Materials and Structures, 4- 2, p. 245-262, 2009

[Chr 10] Chrysochoos A., Huon V., Jourdan F., Muracciole J.-M., Peyroux R. and WAtTRISSE B., " Use of Full-Field DIC \& IRT Measurements for the Thermomechanical Analysis of Material Behaviour ", Strain, 46, pp. 117-130, 2010.

[Col 67] Coleman B,. Gurtin, M., " Thermodynamics with internal state variables ", The Journal of Chem. Phys., vol.47, (2), p. 597-613, 1967.

[Deg 96] Degiovanni A., Batsale J.C., Maillet D., " Mesure de la diffusivité longitudinale de matériaux anisotropes - Panorama des techniques développées au LEMTA ", Rev. Gen de Thermique, 35, pp. 141-147, 1996.

[Dou 09] Doudard C., CAlloch S., " Influence of hardening type on self-heating of metallic materials under cyclic loadings at low amplitude ", Eur. J. of Mechanics, 28-2, pp. 233-240, 2009.

[Fre 07] FrÉmond M., " Non-smooth Thermomechanics ", Berlin, Springer, 2002.

[Ger 83] Germain P., Nguyen Q. S, SuQuet P.," Continuum Thermomechanics », J. Appl. Mech., vol.50, (4B), pp. 1010-1020, 1983.

[FUD 02] Fudym O., BATSAle J.C., LeCOnTE D., " A seminumerical approach for heat diffusion in heterogeneous media, One extension of the analytical quadrupole method ", Numerical Heat Transfer, Part B, 42, pp. 325-348, 2002.

[FUD 04] Fudym O., LADEvie B., BATSALE J.C., " Heat diffusion at the boundary of stratified media, Homogenized temperature field and thermal constriction ", Int J. of Heat and Mass Transfer, vol. 47, pp. 2437-2447, 2004.

[Fud 05] Fudym O., Battaglia J.L., Batsale J.C., " Measurement of 
thermophysical properties in semi-infinite media by random heating and fractional model identification ", Rev. of Sci. Instrum., 76, (2), pp.1-10, 2005.

[FUD 07] Fudym O., Batsale J.C., Battaglia J.L., " Thermophysical properties mapping in semi-infinite longitudinally cracked plates by temperature image processing ", Inverse Problem In Engineering,15, (2), pp.163-176.

[GAU ] GaUSSORgues G., " La Thermographie Infrarouge - Principes, Technologie, Applications ", 4ème Édition, Tec \& Doc Lavoisier, 1999.

[Gus 91] Gustafson SE., " Transient plane source techniques for thermal conductivity and thermal diffusivity measurements of solid materials ", Rev. of Sci. Instrum., 62, 797, 1991.

[Hal 75] Halphen B., NGUYen Q. S., " Sur les matériaux standards généralisés ", Journal de Mécanique, vol. 14, (1), pp.39-63 ; 1975.

[Hay 96] Hay B., Filz J.R., BATSAle J.C , " Mesure de diffusivité thermique par méthode flash ", Techniques de l'ingénieur, R 2 955, pp.1-12, 2004.

[Hon 05] Honorat V., Moreau S., Muracciole J-M., B. Wattrisse B., Chrysochoos A., " Calorimetric analysis of polymer behaviour using a pixel calibration of an IRFPA camera ", Int J. on Quantitative IR Thermography, 2, (2), pp.153-172, 2005.

[Kra 04] Krapez J.C., Spagnolo L., FrieB M., Maier H.P. Netter F., " Measurement of in-plane diffusivity in non-homogeneous slabs by applying flash thermography ", Int. J. of Therm. Sci., 43, pp. 967-977, 2004.

[Lep 96] Lepoutre F., Lefebvre J., Lhermitte T., Ainouch L., Delpech P., Forge P., HiRCHI S., Joulaud J.L., " Mesures thermiques microscopiques ", Rev. Gen., Therm., 413, (35), pp 344-354, 1996.

[Mai 00] Maillet D., Andre S., Batsale J.C., Degiovanni A., Moyne C., " Thermal quadrupoles- Solving the heat equation through integral transforms ", J. Wiley - Chichester- 2000.

[Mur 08] Muracciole JM, Wattrisse B, Chrysochoos A, " Energy Balance of a Semicrystalline Polymer During Local Plastic Deformation ", Strain, 44-6, p 468-474, 2008.

[Paj 89] Pajani D., " Mesure Par Thermographie Infrarouge", Editeur ADD, EAN13 9782950417107, 1989.

[PAP 97] PAPINI F., Gallet P., " Thermographie infrarouge - Image et mesure ", Masson Ed., 1997.

[Pey 98] Peyroux R., Chrysochoos A., Licht C., Löbel M., " Thermomechanical couplings and pseudoelasticity of shape memory alloys ", Int. J. Engng Sci., 36, (4), p.489-509, 1998.

[Phi 95] Philippi I., Batsale J.C., Maillet D., Degiovanni A., " Measurement of thermal diffusivity trough processing of infrared images ", Rev. Sci. Instrum., 66, pp. 182-192, 1995.

[Pin 04] Pina V., Ranc N., Wagner D., Herve P., Sutter G., Philippon S., 
30 IR thermographic imaging

" Étude des comportements statique et dynamique de matériaux métalliques par pyrométrie ultraviolet, visible ou infrarouge ", Photonique, 16, pp. 1-4, 2006.

[Pre 07] Press W.H., Teukolsky S.A., Wetterling W.T., Flannery B.P., "Numerical recipes, The art of scientific computing ", $3^{\text {rd }}$ edition, Cambridge University Press, 2007.

[PRO 04] PRON H., BISSIEUX C., " 3D thermal modelling applied to stress-induced anisotropy of thermal conductivity", Int. J. Therm. Sci., Vol 43/12 (2004) pp. 1161-1169.

[Ran 08] Ranc N., Taravella L., Pina V., Hervé P., " Temperature field measurement in titanium alloy during high strain rate loading - adiabatic shear bands phenomenon ", Mechanics of Materials , 40, (4-5), pp. 255-270, 2008.

[SAt 07] SATHON N., Dulieu-Barton J.M., " Evaluation of sub-surface stresses using thermoelastic stress analysis ", Applied Mechanics and Materials, 7-8, pp.153-158, 2007.

[SuT 07] SutTer G., N. RanC N., " Temperature fields in a chip during high-speed orthogonal cutting - An experimental investigation ", Int. J. of Machine Tools \& Manufacture, 47, pp. 1507-1517, 2007.

[Tho 53] Thomson W. (LoRd Kelvin), " On the thermoelastic and thermomagnetic properties of matter", Trans. Roy. Soc. Edinb., 20, (161), pp.57-77, 1853. 